\title{
APENDICITE CRÔNICA E APENDICITE RECORRENTE. ARTIGO DE REVISÃO E APRESENTAÇÃO DE CASUÍSTICA
}

\author{
CHRONIC AND RECURRENT APPENDICITIS. REVIEW ARTICLE AND CASES REPORT
}

José Joaquim Ribeiro da Rocha, Francisco Aprilli \& Omar Féres

Docentes da Disciplina de Coloproctologia do Departamento de Cirurgia e Anatomia da Faculdade de Medicina de Ribeirão Preto-USP Correspondência: Prof.Dr. José Joaquim Ribeiro da Rocha. Departamento de Cirurgia e Anatomia. Hospital das Clínicas de Ribeirão Preto, 9ํandar, CEP 14.049-900 - Ribeirão Preto-SP

ROCHA JJR; APRILLI F \& FÉRES O. Apendicite crônica e apendicite recorrente. Artigo de revisão e apresentação de casuística. Medicina, Ribeirão Preto, 34, 292-296, jul./dez. 2001.

RESUMO: A apendicite crônica e a apendicite recorrente são condições patológicas que diferem da apendicite aguda, a afecção mais comum do apêndice vermiforme.

O propósito deste artigo é decifrar, com critério, os aspectos clínicos, o diagnóstico e a histopatologia da apendicite crônica e da apendicite recorrente.

Os dados da literatura e as experiências, clínica e cirúrgica, dos autores, demonstradas dentre 10 pacientes com idade média entre 40 e 45 anos, com distribuição similar quanto ao sexo, sendo seis pacientes com apendicite recorrente e quatro com apendicite crônica, permitem concluir que a apendicite crônica e a recorrente já não devem ser mais uma controvérsia ou dúvida, mas um fato inequívoco, de importância clínica e acadêmica, cujos conceitos, por isso mesmo, merecem difusão na literatura científica e nas escolas médicas.

UNITERMOS: Apendicite Crônica. Apendicite Recorrente. Apendicite Aguda.

\section{INTRODUÇÃ̃}

O diagnóstico de apendicite crônica e recorrente continua controverso para muitos. O ensino de cirurgia ressalta que o apêndice pode apresentar uma resposta inflamatória com uma única evolução patológica, chamada apendicite aguda.

Entretanto, há pacientes com evoluções clínicas distintas, caracterizadas por surtos recorrentes de apendicite aguda ou dor crônica no quadrante inferior direito, que sugerem outras entidades do apêndice, conhecidas como apendicite recorrente e apendicite crônica, respectivamente ${ }^{(1 / 5)}$.

A despeito da vasta experiência clínica com apendicite aguda, o diagnóstico da apendicite crônica e o da recorrente ainda não são aceitos por unanimidade. No entanto, nos últimos anos, várias referências confiáveis têm relatado séries de pacientes com crises repetitivas de apendicite aguda e de apendicite crônica de longa duração ${ }^{(1,5)}$.

Esses pacientes evoluem com crises de dor abdominal recorrente ou dor abdominal crônica, nesses casos, o diagnóstico de apendicite não foi definido pela característica da periodicidade da dor, embora a apendicite deva ser considerada no diagnóstico diferencial da dor abdominal, crônica e recorrente, do quadrante inferior do abdômen.

Neste trabalho, foi realizada uma revisão da literatura e relatada a experiência dos autores com dez pacientes portadores de apendicite crônica e apendi- 
cite recorrente. São enfatizados os achados clínicos, os exames para diagnóstico, os aspectos cirúrgicos e histopatológicos.

\section{ASPECTOS CLÍNICOS E PATOLÓgICOS}

Apendicite aguda é a doença mais freqüente do apêndice vermiforme e a operação é a conduta de primeira escolha para o seu tratamento. Esses conceitos são conhecidos desde Fitz, em $1886^{(6)}$. Sua incidência é mais freqüente em jovens em idade escolar e adolescentes, predominantemente do sexo masculino. Pode manifestar-se em diferentes fases: catarral, supurativa, gangrenosa e hiperplásica. A depender de cada caso, há presença de neutrófilos, pus, ulcerações, gangrena e peritonite local ou difusa, se ocorreu rotura do órgão ${ }^{(7)}$.

O diagnóstico de apendicite aguda é eminentemente clínico, sendo feito com base nos sintomas e sinais clínicos mais freqüentes: dor epigástrica e periumbilical, náuseas e/ou vômitos, dor na fossa ilíaca direita, febre, sinais de Blumberg, Rovsing, Chutro e do obturador, entre outros ${ }^{(7)}$.

A apendicite crônica e a recorrente são, por sua vez, entidades distintas da apendicite aguda bem como de outros processos inflamatórios do apêndice, envolvendo diferentes mecanismos patológicos, como por exemplo, a mucocele e o divertículo apendicular.

A etiopatogenia da apendicite crônica e a da recorrente ainda não estão bem compreendidas. Um conceito bem aceito é o mecanismo de obstrução intermitente ou parcial do lúmen apendicular, como acontece na hiperplasia mucosa, levando à mucocele. Quando a distensão se torna máxima, a pressão dentro do lúmen apendicular força a saída da secreção mucosa através do orifício apendicular para dentro do ceco, com conseqüente melhora dos sintomas ${ }^{(8,9)}$.

O critério para diagnóstico de apendicite recorrente foi constituído pela apresentação de crises recorrentes de dor em quadrante inferior direito do abdômen com ou sem outros sintomas e sinais sugestivos de apendicite aguda, e que culminaram em apendicectomia, pela histopatologia, que demonstrou processo inflamatório agudo, do apêndice e pelo desaparecimento dos sintomas após a cirurgia.

O critério para o diagnóstico de apendicite crônica foi constituído pela dor crônica, (acima de um mês), em quadrante inferior direito, pelo achado cirúrgico e histopatológico de processo inflamatório crôni- co, com ou sem fibrose e, por fim, pelo alívio dos sintomas após apendicectomia.

A maioria desses eventos tem o seu diagnóstico impreciso, e eles são rotulados como gastroenterites, aderências pélvicas, doença de Cröhn, oclusão intestinal, diverticulites ou mesmo dor abdominal de etiologia desconhecida, e, não raramente, com uma longa história clínica.

Em uma revisão de 1000 apendicectomias, num período de 10 anos, encontraram-se $0,8 \%$ de apendicites crônicas e $9 \%$ de apendicites recorrentes ${ }^{(10)}$. Outra revisão, de 205 pacientes operados, revela uma incidência semelhante de $10 \%$ de apendicites recorrentes e de $1,5 \%$ de apendicites crônicas ${ }^{(3)}$.

Dados da literatura e casuística dos autores deste trabalho demonstram uma distribuição similar quanto ao sexo, porém com idade média entre 40-45 anos, portanto, acima daquela encontrada, nos pacientes com apendicite aguda.

A histopatologia da apendicite recorrente é semelhante àquela dos pacientes com apendicite aguda, processo inflamatório agudo, exsudativo, fibrinoleucocitário e ulcerações flegmonosas. Nos casos de apendicite crônica, os achados histopatológicos são de inflamação crônica, com presença de linfócitos e eosinófilos dentro da parede celular, geralmente, associada à fibrose, com ou sem obliteração luminal ${ }^{(11)}$.

Alguns dados do estudo retrospectivo de dez pacientes, com idade média de 42 anos e duração média dos sintomas de 22,7 meses, todos operados e seguidos pelo autor deste trabalho, estão dispostos na Tabela I.

\section{EXAMES COMPLEMENTARES}

Os pacientes com dor abdominal crônica, habitualmente são extensivamente avaliados através de exames de imagem como, ultra-som, tomografia computadorizada e enema de bário, entre outros.

Quando a dor é localizada no quadrante inferior direito, o enema de bário é freqüentemente solicitado. Nessas ocasiões, em que não há suspeita de doença apendicular, em cerca de 5 a $10 \%$ dos casos, o apêndice não será contrastado e, por conseguinte, em 90 a 95\%, os apêndices estarão preenchidos pelo bário $^{(12,13)}$.

Entretanto, é comum a presença de alterações radiográficas do apêndice, nos casos de dor crônica do quadrante inferior direito do abdômen, em que a 


\begin{tabular}{|c|c|c|c|c|c|}
\hline Pacientes & $\begin{array}{l}\text { Faixa } \\
\text { etária/Sexo }\end{array}$ & $\begin{array}{l}\text { Duração dos } \\
\text { sintomas }\end{array}$ & Diagnóstico inicial & Histopatologia & Diagnóstico final \\
\hline 1 & $36 / \mathrm{m}$ & 4 anos & Gastroenterite & $\begin{array}{l}\text { Apêndice com foco ulcerativo + } \\
\text { depósitos fibrinoleucocitários }\end{array}$ & $\begin{array}{l}\text { Apendicite } \\
\text { recorrente }\end{array}$ \\
\hline 2 & $42 / f$ & 1 ano & Aderências pélvicas & $\begin{array}{l}\text { Apêndice escleroatrófico c/ } \\
\text { obliteração fibrosa luminal }\end{array}$ & Apendicite crônica \\
\hline 3 & $42 / m$ & 7 meses & Doença de Cröhn & $\begin{array}{l}\text { Apendicite aguda ulcero- } \\
\text { flegmonosa fibrinopurulenta }\end{array}$ & $\begin{array}{l}\text { Apendicite } \\
\text { recorrente }\end{array}$ \\
\hline 4 & $25 / f$ & 3 anos & Gastrite & $\begin{array}{l}\text { Apendicite aguda ulcerativa, } \\
\text { focal, com exsudato fibrino- } \\
\text { leucocitário }\end{array}$ & $\begin{array}{l}\text { Apendicite } \\
\text { recorrente }\end{array}$ \\
\hline 5 & $40 / f$ & 1 ano & Aderências pélvicas & $\begin{array}{l}\text { Infiltrado inflamatório, crônico, } \\
\text { com áreas de ulceração, parede } \\
\text { espessa e fibrosa }\end{array}$ & Apendicite crônica \\
\hline 6 & $45 / m$ & 6 meses & $\begin{array}{l}\text { Suboclusão } \\
\text { intestinal }\end{array}$ & $\begin{array}{l}\text { Apêndice com ulceração + fibrina } \\
+ \text { + infiltrado leucocitário, } \\
\text { neutrofílico }\end{array}$ & $\begin{array}{l}\text { Apendicite } \\
\text { recorrente }\end{array}$ \\
\hline 7 & $57 / f$ & 8 meses & Gastroenterite & $\begin{array}{l}\text { Apêndice escleroatrófico, fibrose } \\
\text { em toda a parede. Infiltrado } \\
\text { inflamatório com eosinófilos }\end{array}$ & Apendicite crônica \\
\hline 8 & $40 / m$ & 3 anos & $\begin{array}{l}\text { Dor abdominal a } \\
\text { esclarecer }\end{array}$ & $\begin{array}{l}\text { Apendicite aguda fibrino- } \\
\text { purulenta e flegmonosa }\end{array}$ & $\begin{array}{l}\text { Apendicite } \\
\text { recorrente }\end{array}$ \\
\hline 9 & $40 / m$ & 5 anos & $\begin{array}{l}\text { Dor abdominal a } \\
\text { esclarecer }+ \\
\text { gastroenterite }\end{array}$ & $\begin{array}{l}\text { Apendicite aguda, supurativa, } \\
\text { com exsudato fibrinoleucocitário }\end{array}$ & $\begin{array}{l}\text { Apendicite } \\
\text { recorrente }\end{array}$ \\
\hline 10 & $54 / \mathrm{m}$ & 2 meses & Diverticulite & Processo inflamatório crônico & Apendicite crônica \\
\hline
\end{tabular}

exploração cirúrgica demonstra doença apendicular ${ }^{(1)}$. Os sinais radiográficos, descritos na apendicite aguda, quando se realiza o enema de bário, incluem: falha de enchimento, parcial ou completa, do lúmen apendicular, compressão extrínseca do ceco, semelhante ao número três invertido e irregularidade de contorno do ceco e/ou íleo terminal ${ }^{(14)}$.

O enema de bário é, portanto, considerado um exame útil no diagnóstico da apendicite aguda, quando realizado ${ }^{(15,16,17)}$. Da mesma maneira o é em pacientes com apendicite crônica e apendicite recorrente, que, quando submetidos a esse exame, revelam achados radiográficos semelhantes aos da apendicite $\operatorname{aguda}^{(15)}$. Em nossa experiência, oito pacientes foram submetidos ao enema opaco e todos apresentaram alterações do ceco e/ou apêndice, sendo a falha de enchimento do lúmen apendicular o achado mais comum (Figuras 1, 2 e 3).

Uma vez que as alterações anatômicas e morfológicas do apêndice e adjacências são pouco proe- minentes, nos casos de apendicite crônica e apendicite recorrente, a ultra-sonografia e a tomografia computadorizada são pouco sensíveis nessas situações, podendo ser úteis nos casos de apendicite recorrente com coleções intraperitoneais.

\section{TRATAMENTO}

O uso de analgésicos, antitérmicos, antiinflamatórios e antibióticos, pode melhorar as condições clínicas e os sintomas, transitoriamente; posteriormente esses pacientes voltarão a apresentar dor abdominal.

O único tratamento com propósito curativo é a abordagem cirúrgica com apendicectomia, seja por via aberta ou por via laparoscópica.

Devido ao caráter de cronicidade e recorrência dessas situações, é freqüente encontrar apêndices espessos, fibrosos, aderidos ao epíploo, íleo, ceco, parede abdominal, anexos uterinos e alças de intestino delgado. 


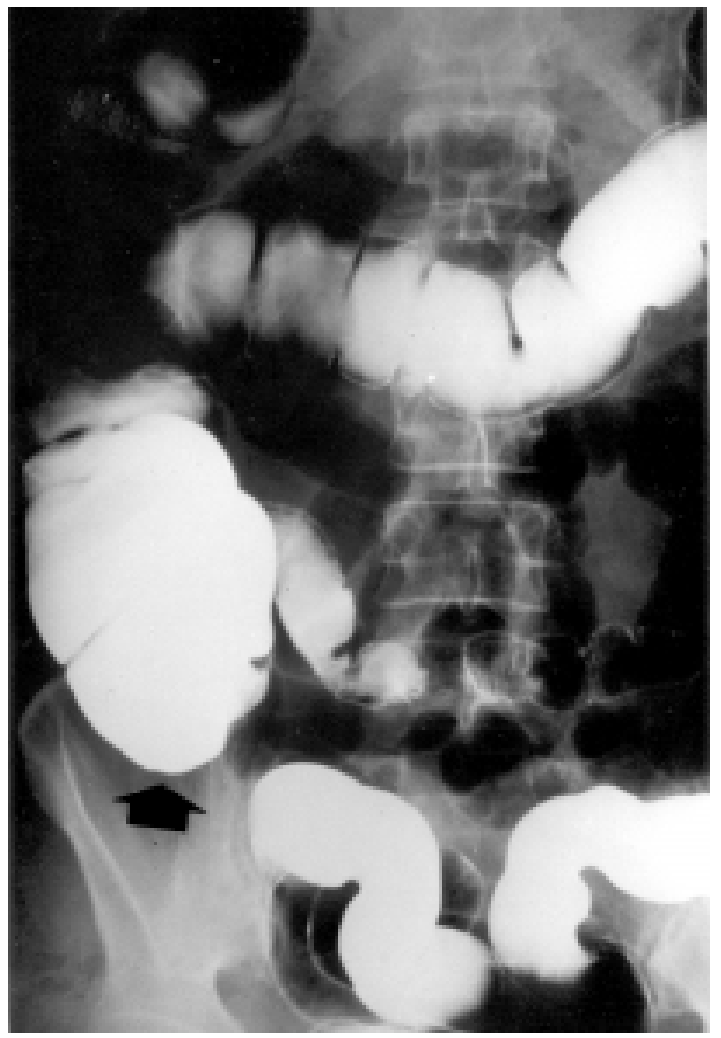

Figura 1: Enema opaco. Não há contrastação de apêndice (a seta indica o ceco).

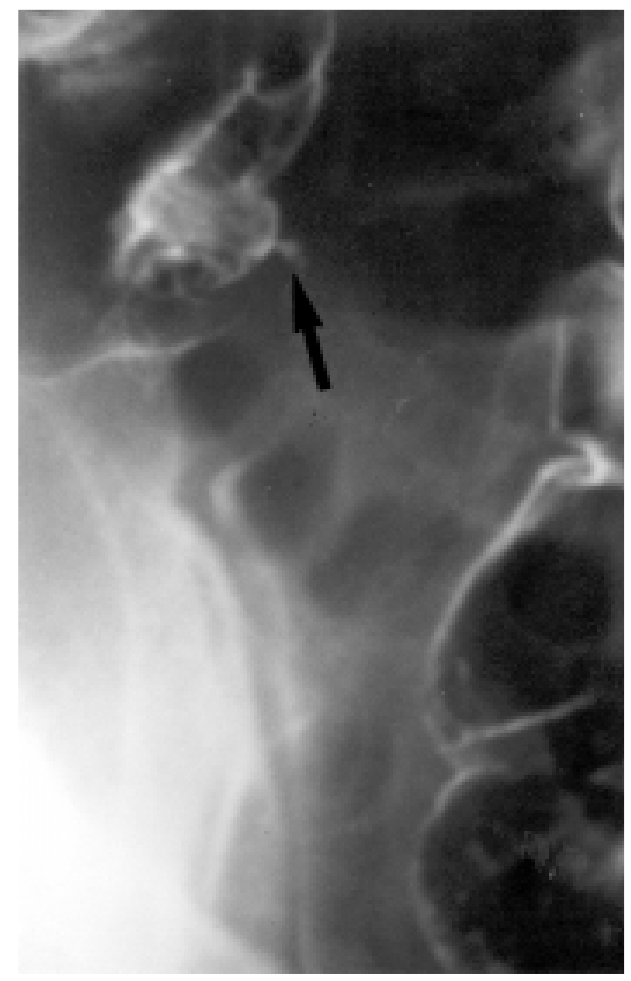

Figura 2: Enema opaco. Falha de enchimento do segmento distal do apêndice (a seta indica a base do apêndice contrastado).

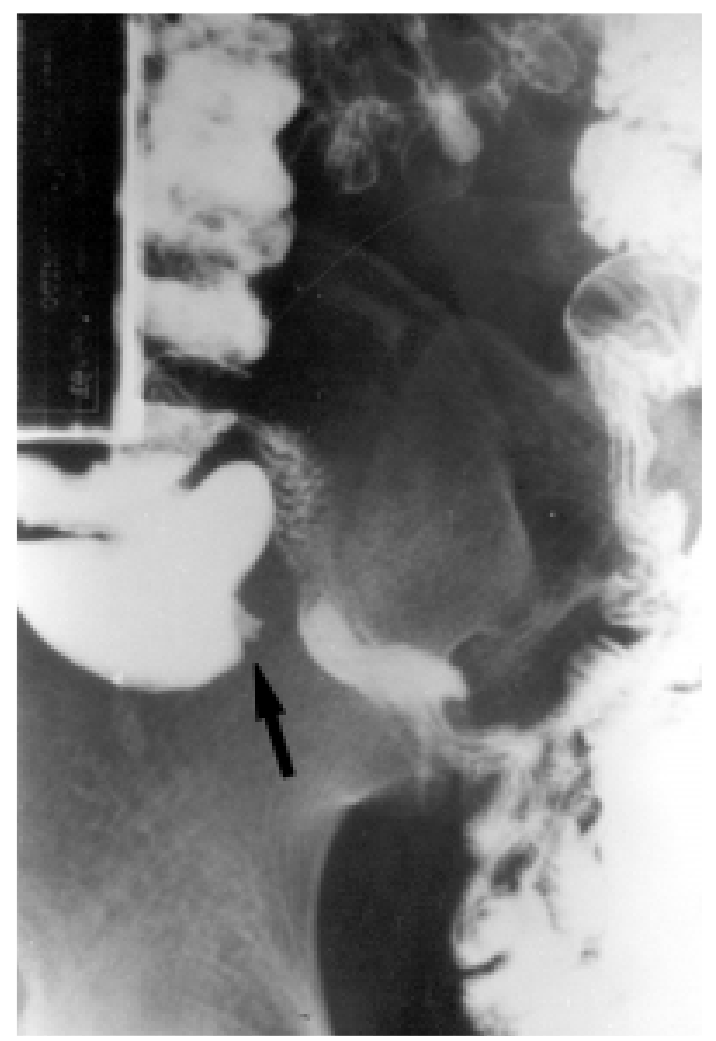

Figura 3. Enema opaco. Irregularidade do contorno do ceco (seta indica a base do apêndice, contrastada levemente)

\section{CONSIDERAÇÕES FINAIS}

De maneira pouco convincente, o diagnóstico de apendicite crônica passou a ser utilizado para explicar situações de dor abdominal crônica sem causa aparente $^{(2)}$ Desse modo, a apendicectomia tornou-se um dos mais comuns procedimentos cirúrgicos nos primórdios do século 20 , com pouca contestação ${ }^{(15)}$, como demonstra um estudo de revisão de 50.000 apêndices operados entre 1924 e 1955, dentre os quais um terço correspondia à apendicite crônica e aproximadamente $14 \%$ à apendicite aguda ${ }^{(18)}$. Em 1940, uma revisão de 385 pacientes, submetidos à apendicectomia por dor abdominal crônica ou recorrente, 255 não tinham historia clínica compatível com apendicite; desses, somente dois foram curados da dor e 60 ficaram em condições piores. Por outro lado, de 130 pacientes com história clínica característica de apendicite aguda ou recorrente, $92 \%$ foram curados ou melhoraram no pós-operatório . Deduz-se por esses trabalhos que o desconhecimento quer seja da fisiopatologia ou dos aspectos clínicos da apendicite crônica e da apendicite recorrente leva a maus resultados nos pósoperatório $^{(19,20)}$. 
Para finalizar, é importante salientar e ilustrar a experiência clínica com dez pacientes operados no período de 1991 a 1998 no Hospital das Clínicas de Riberão Preto e em clínica privada. As características clínicas do diagnóstico e os aspectos histopatológicos desses pacientes estão na Tabela I.

Baseando-se em nossos resultados e naqueles relatos da literatura, consideramos que a apendicite crônica e a recorrente já não devem ser uma contro- vérsia ou dúvida mas um fato real, de importâncias clínica e acadêmica. Podemos afirmar que constituem doenças distintas e bem caracterizadas e devem ser lembradas no diagnóstico diferencial, nos casos de dor de longa duração no quadrante inferior direito, crônica ou recorrente. Portanto, informações acerca dessas entidades devem ser amplamente divulgadas na literatura científica e nas escolas médicas.

ROCHA JJR; APRILLI F \& FÉRES O. Chronic and recurrent appendicitis. Review article and cases report. Medicina, Ribeirão Preto, 34: 292-296, july/dec. 2001.

ABSTRACT: Chronic and recurrent appendicitis are pathologic conditions that differ from acute appendicitis, the most common affection of the vermiform appendix.

The objective of the present study was to describe in detail the clinical features, diagnosis and pathology of those conditions.

UNITERMS: Chronic Appendicitis. Recurrent Appendicitis. Acute Appendicitis.

\section{REFERÊNCIAS BIBLIOGRÁFICAS}

1 - GROSSMANN Jr E B. Chronic appendicitis. Surg Gynecol Obstet 146: 596-598, 1978.

2 - SAVRIN RA; CLAUSEN K; MARTIN EW \& COOPERMAN M. Chronic and recurrent appendicitis. Am J Surg 137: 355357, 1979.

3 - CRABBE MM; NORWOOD SH; ROBERTSON HD \& SILVA JS. Recurrent and chronic appendicitis . Surg Gynecol Obstet 163: 11-13, 1986.

4 - HAWES AS \& WHALLEN FG. Recurrent and chronic appendicitis: the other inflammatory conditions of the appendix. Am Surg 60: 217-219, 1994

5 - MATTEI P, SOLA JE \& YEA JC. Chronic and recurrent appendicitis are uncommon entities often misdiagnosed. J Am Coll Surg 178: 385-389, 1994.

6 - FITZ RH. Perforating inflammation of the vermiform appendix with special reference to early diagnosis and treatment. Am J Med 92: 321-346, 1886.

7 - OKANO N E \& VARGAS EC. Acute appendicitis. Medicina, Ribeirão Preto, 28: 676-680, 1995.

8 - LEE AW; BELL RM; GRIFFEN WO \& HAGIHARA PF. Recurrent appendiceal colic. Surg Gynecol Obstet 162: 2124, 1985.

9 - HOWER MJ \& BRAVER JM. Recurrent appendicitis: reexamination of a controversial disease. Gastrointest Radiol 4: 295-301, 1979.

10 - DYMOCK RB. Pathological changes in the appendix: a review of 1000 cases. Pathology 9: 331-339, 1977.

11 - SAKOVER RP \& DELFAVE RL. Frenquencly of visualization of the normal appendix with the barium enema examination. Am J Roentgenol 121: 312-317, 1974.
12 - RAJOGOPALAN AE; MASON JH; KENNEDY M \& PAWLIKOWSKI J. The value of the barium enema in the diagnosis of acute appendicitis. Arch Surg 112: 531-533, 1977.

13 - SETER CS. The use of barium enema in the diagnosis of acute appendicecal disease: a new radiographic sign. Clin Radiol 19: 410-415, 1968.

14 - SMITH DE; KIRCHMER NA \& STEWART DR. Use of the barium enema in the diagnosis of acute appendicitis and its complictions. Am J Surg 138: 829-834, 1979.

15 - COLLINS DC. A study of 50.000 specimens of the human vermiform appendix. Surg Gynecol Obstet 101: 437-445, 1955.

16 - FEE HJ; JONES PD; KADELL B \& O ' CONNELL, TX. Radiologic diagnosis of appendicitis. Arch Surg 112: 742-744, 1977.

17 - SEIDMAN JD; ANDERSEN DK \& ULRICH S. Recurrent abdominal pain due to chronic appendicecal disease. South Med J 84: 913-916, 1991.

18 - ALVAREZ W. When should one operate for "chronic appendicitis" JAMA 114: 1301, 1940.

19 - CARNET JB \& BOLES RS. Fallacies concerning chronic appendicits. JAMA 91: 1679-1683, 1928.

20 - LEWIS FR; HOLCROFT JW; BOEY J \& DUNPHY E. apendicitis: a critical review of diagnosis and treatment in 1000 cases. Arch Surg 110: 677-684, 1975.

Recebido para publicação em 12/02/2001

Aprovado para publicação em 10/09/2001 